\title{
PROMOÇÃO DA SAÚDE DE PESSOAS COM DIABETES MELLITUS NO CUIDADO EDUCATIVO PREVENTIVO DO PÉ-DIABÉTICO ${ }^{1}$
}

\author{
HEALTH PROMOTION OF PEOPLE WITH DIABETES MELLITUS \\ REGARDING DIABETIC FOOT CARE
}

\section{PROMOCIÓN DE SALUD DE PERSONAS CON DIABETES MELLITUS EN EL CUIDADO PREVENTIVO DEL PIE DIABÉTICO}

\author{
LuZia Wilma Santana da Silva* \\ JAREDE SOUZA Silva \\ Camila Fabiana Rossi Souarcini ${ }^{* * *}$ \\ Fabiana Galvão SoUZA ${ }^{* * *}$ \\ VALÉria dos Santos Ribeiro***** \\ Déborah Ferreira GonÇALVes ${ }^{* * * * *}$
}

\begin{abstract}
RESUMO
Objetivo: Conhecer os resultados das ações intervencionistas de educação em saúde à prevenção do pé-diabético. Método: Estudo de método misto, avaliativo-interpretativo na abordagem pesquisa-ação na primeira etapa, oriundo de um projeto guarda-chuva, desenvolvido na Universidade Estadual do Sudoeste da Bahia, Jequié, BA, aprovado pelo Comitê de Ética da citada universidade. A amostra foi composta por 13 idosas. Os instrumentos: um questionário estruturado; um roteiro sistematizado para observação de campo e os registros do conteúdo programático da abordagem educativo-problematizadora do projeto guarda-chuva. Os dados foram analisados na perspectiva interpretativo-compreensica sugerida por Miles e Huberman. Na etapa quantitativa para análise dos dados, fez-se uso da estatística descritiva. Resultados: A estratégia pesquisa-ação foi potencializadora da abordagem educação em saúde ao cuidado de si das participantes, na adoção de medidas protetivas no cuidado com os pés. Conclusão: Mostra-se como fundamental a estratégia de metodologia proximal em práticas que
\end{abstract}

\footnotetext{
${ }^{1}$ Estudo faz parte do projeto guarda-chuva: “Educação em saúde no enlace diagnóstico preventivo do pé-diabético”, do Núcleo interdisciplinar de estudos e extensão em cuidados à saúde da família em convibilidade com doenças crônicas - NIEFAM /DS II-UESB. Foi desenvolvido com apoio da PROEX/GEAC-UESB. Bolsa de Iniciação científica PIC/UESB.

* Enfermeira. Professora MS, Ph.D. em Enfermagem, Titular do Depart ${ }^{\circ}$. de Saúde II/ Universidade Estadual do Sudoeste da Bahia (UESB). Líder do Grupo de Pesquisa Interdisciplinar de Estudos em Ciências da Saúde e Sociedade. Linha de pesquisa: Família em seu ciclo vital. Coord. do Núcleo Interdisciplinar de Estudos e Extensão em Cuidados à Saúde da Família em Convibilidade com Doenças Crônicas (NIEFAM). Jequié, BA, Brasil. Email: luziawilma@yahoo.com.br

** Graduada em enfermagem pela UESB. Bolsista de Iniciação Científica PIC-UESB, período ago2013-jul2014. Membro do NIEFAM. Linha de Pesquisa: Família em seu ciclo vital. Jequié, BA, Brasil. Email: jarede_souza@hotmail.com

${ }^{* *}$ Mestre em Educação Física. Doutoranda em Educ. Física/UFSC. Profa. Assistente do Depart ${ }^{\circ}$. de Saúde I/UESB. Membro do Grupo de Pesquisa Interdisciplinar de Estudos em Ciências da Saúde e Sociedade do NIEFAM. Linha de pesquisa: Família em seu ciclo vital. Email: csquarcini@gmail.com

${ }^{* * * *}$ Graduada em enfermagem pela UESB. Bolsista de Iniciação Científica PIBIC-CNPq, período ago2013-jul2014. Membro NIEFAM. Linha de Pesquisa: Família em seu ciclo vital. Jequié, BA, Brasil. Email: faby_jq@hotmail.com

${ }^{* * * * *}$ Graduada em Enfermagem pela UESB. Bolsista de Extensão do NIEFAM/PROEX-GEAC. Linha de Pesquisa: Família em seu ciclo vital. Jequié, BA, Brasil. Email:vavalribeiro@gmail.com

${ }_{* * * * * *}$ Graduada em Enfermagem pela UESB. Bolsista de Extensão do NIEFAM/PROEX-GEAC, período ago2013-jul2014. Linha de Pesquisa: Família em seu ciclo vital. Jequié, BA, Brasil. Email: binhafgoncalves@hotmail.com
} 
aliem educação em saúde, treinamento e sistematização do autocuidado, de modo à reorientação do modelo assistencial, potencializador dos cuidados em Enfermagem.

Palavras chave: Pé-diabético, promoção da saúde, cuidados de enfermagem.

\begin{abstract}
Objective: To obtain results of intervention actions regarding health education in the prevention of diabetic foot. Methods: An evaluative-interpretive mixed method study with an action research approach in the first stage, extracted from a macro-project at the State University of Southwest Bahia, Jequié, BA, approved by the Ethics Committee of that institution. The sample was composed of 13 elderly (female); the instruments were a structured questionnaire, a systematized guide for field observation and records from the aforementioned macro-project's content program, which has a problematization approach. Data were analyzed in the interpretative-comprehensive perspective suggested by Miles and Huberman. During the qualitative stage, data was analyzed through descriptive statistics. Results: The action research strategy strengthened the health education approach of self-care regarding the adoption of preventive foot care. Conclusion: It shows the importance of the proximal methodology strategy in practices that combine health education, training and systematization of self-care in order to redirect the care model, which enhances nursing care.
\end{abstract}

Key words: Diabetic foot, health promotion, nursing care.

\title{
RESUMEN
}

Objetivo: Conocer los resultados de las acciones intervencionistas de la educación en salud del pie diabético. Material y método: Estudio de métodos mixtos evaluativo-interpretativo con un enfoque de investigaciónacción en la primera etapa, parte de un macro-proyecto de la Universidad Estatal del Suroeste de Bahía, Jequié, BA, aprobado por el Comité de Ética de dicha institución. La muestra fue compuesta por 13 ancianas. Los instrumentos fueron un cuestionario estructurado, un guión sistematizado para la observación de campo y los registros del contenido programático del abordaje educativo-problematizador del referido macro-proyecto. Los datos fueron analizados en la perspectiva interpretativa-exhaustiva sugerida por Miles y Huberman. En la etapa cuantitativa, para el análisis de los datos, se utilizó estadística descriptiva. Resultados: La estrategia investigaciónacción fue potencializadora del abordaje de la educación en salud al autocuidado de las participantes en la adopción de medidas protectoras en el cuidado de los pies. Conclusión: Se verifica que es fundamental la metodología proximal en prácticas que pacten educación en salud, el entrenamiento y la sistematización del autocuidado con el fin de reorientar el modelo de atención, potenciador del cuidado de enfermería.

Palabras clave: Pie diabético, promoción de salud, atención de enfermería.

Fecha recepción: 12/08/14 Fecha aceptación: 15/03/16

\section{INTRODUÇÃO}

Vivemos em um momento histórico, no Brasil, em que epidemiologicamente doenças de natureza crônica alavancam os índices de morbimortalidade nunca antes imagináveis, a exemplo o Diabetes Mellitus (DM), sendo também uma doença que eleva a cifra de epidemia mundial. Trata-se de um distúrbio metabólico que acomete as pessoas de forma progressiva e degenerativa com impacto no processo de viver humano (1).

$\mathrm{O}$ DM reverte-se em um sério desafio à saúde mundial, sobretudo, por se estar vivendo mais e viver mais, ainda representa um estar exposto as alterações de ordem morfofisiológica, susceptível à ocorrência de no- 
sologia de natureza crônica. Outros fatores, no entanto, vêm somar-se a esta evidência, a exemplo a crescente urbanização, sedentarismo, consumo inadequado de alimentos que proporcionam aumento dos níveis glicêmicos, obesidade, dentre outros (2).

Dados da Organização Mundial de Saúde estimavam que no ano de 2000 haviam 177 milhões de pessoa com DM em todo mundo, com expectativa para o ano 2025 de 350 milhões (2). Esta expectativa não encontra aderência aos estudos epidemiológicos atuais, nos quais a cada momento novos números somam-se a problemática, já em 2009 as estimativas para 2025 eram de que $5,4 \%$ da população mundial teria $\mathrm{DM}$, sendo a prevalência maior para os países em desenvolvimento (3).

Outro aspecto a considerar é a associação DM e Hipertensão Arterial Sistêmica (HAS). Doenças que comumente acometem ao mesmo tempo as pessoas e estão entre as causas de maior incidência de hospitalização e mortalidade no Brasil (4). O DM, em particular, pelas amputações de membros inferiores por neuropatia diabética (ND) ou pé-diabético.

$\mathrm{O}$ DM é definido como um conjunto de diferentes distúrbios metabólicos, que em comum culminam em hiperglicemia como resultado de alterações pancreáticas e da liberação da insulina ou ainda pelas duas causas, de modo que as pessoas possuem grande possibilidade de desenvolverem sérias complicações. Dentre elas, àquelas que afetam o sistema circulatório, endócrino, renal, neurológico e outros, sendo uma das mais severas e comuns à ND (5). Neste particular, centra-se o olhar deste estudo.

A ND afeta as pessoas em seu processo de viver humano devido às recorrentes intercorrências do DM, e também, o sistema de saúde, pois se trata de um problema que se dá de forma prolongada com alto custo. Neste contexto, trata-se de uma evidência que poderia ser minimizada ou mesmo evitada, se se considerar a relevância da assistência multiprofissional às pessoas com DM (5). É salutar destacar, que qualquer lesão por mais inicial e de pequena extensão que seja, poderá resultar em perdas funcionais, amputações únicas, múltiplas e subsequentes e até a morte (6). Assim, exigindo um olhar multidimensional, por quanto multiprofissional, a não negligência dos cuidados em saúde na atenção primária a pessoa com DM.

Portanto, a avaliação dos pés precisa ser uma estratégia primeira nas consultas em saúde pelos profissionais, em destaque enfermeiros e médicos. Para tanto, estes profissionais precisam estar habilitados para bem saber-fazer o que exige didática para classificar as complicações e os sintomas apresentados no pé-diabético (7). Estas ocorrem devido às alterações nos nervos, podendo incluir sintomas sensitivos como parestesias, sensação de queimação em extremidade, algia local e desequilíbrio; sintomas de ordem motora como atrofia muscular e fraqueza; e autonômicos como, por exemplo, pele com ressecamento excessivo e aumento de transpiração. Outros incluem: fatores como movimento articular limitado, patologias do sistema circulatório periférico, distúrbios do tipo tróficos de pele e distribuição irregular de forças mecânicas sob os pés (6).

A literatura é profícua em estudos sobre os fatores de risco ao pé-diabético, destacando aqueles cujas pessoas são acometidas por ulcerações recursivas por perda de sensibilidade e deformidades; deficiência nas ações de educação em saúde; presença de calos e lesões não ulcerativas; uso de calçados inadequados; tabaco, doenças como HAS e dislipidemia; rebaixamento do nível de acuidade visual; dificuldade de acesso ao sistema de saúde (3).

Do evidenciado, a problemática inquietanos a necessidade de os profissionais de saúde, destacando os da enfermagem, promover ações de educação em saúde que estimulem as capacidades de resiliência ao autocuidado das pessoas sobre enfrentamento do DM. Esse destaque assenta-se na compreensão de que, em sendo uma profissão que se mostra em maior número em contingente humano 
na atenção básica $(\mathrm{AB})$ poderá promover ações educativa-cuidativas ao sujeito do cuidar profissional, que possam reverter a problemática, e, ainda, torná-lo um agente multiplicador de saberes.

Tal conhecimento, ações educativa-cuidativas, deve mediar-se pela relação proximal e sensível de modo a despertar no sujeito do cuidar a ação de corresponsabilização no cuidado de si, e assim, empreender mudanças de ideologias, concepções e comportamentos, cuja finalidade seja aumentar sua autoestima e potencializar seu autocuidado. Trata-se de enveredar pela perspectiva da educação em saúde, objetivando o repensar/agir dos modos de vida do ser-estar, e assim, ao autogerir mudanças de hábitos que possam ser incorporados aos saberes no cuidar de si (5).

Neste direcionamento, no âmbito da ciência Enfermagem, a Teoria do Autocuidado (8) ao considerar a capacidade que o ser humano possui de desempenhar atitudes de cuidar-se contribui ao exercício do cuidado da ciência Enfermagem, corroborando a estratégia de potencialidade da pessoa à efetivação da corresponsabilização com o cuidado de si -O eu interior do cuidado de si transcende o self ao eu social- à manutenção, restabelecimento e promoção de sua saúde na $\mathrm{AB}$ de modo ao controle da glicemia; a inserção em grupos educativos e ao desenvolvimento das capacidades resilientes ao caminho do alcance do sucesso terapêutico, e, por conseguinte, contributiva a redução de amputação de membros inferiores, a qual acomete cerca de 2,8 a 43,9 por 100000 habitantes/ano contexto mundial (9), e com incidência de 13,9 por 100000 habitantes/ano no cenário nacional (10).

Essa problemática nos inquieta ao tempo em que nos motiva a realizar esta pesquisa, cujo objetivo foi conhecer os resultados das ações intervencionistas de educação em saúde à prevenção do pé diabético de pessoas com DM, participantes em um núcleo de pesquisa-extensão em saúde, desenvolvido em uma universidade pública, do interior da Bahia, Brasil.
Como relevância do estudo proporcionar melhores condições de qualidade de saúde tanto à pessoa com DM quanto ao seu sistema familiar de pertencimento bem como potencializá-los para tornarem-se multiplicadores dos saberes sobre o cuidado com os pés. Outro aspecto a considerar, trata-se da formação acadêmica à pesquisa e a inserção social, visto que, este estudo, desenvolveu-se através de bolsas de Iniciação Cientifica e de Extensão no âmbito da ciência Enfermagem, o que foi ao encontro das Leis de Diretrizes e Bases da Educação Brasileira de formar sujeitos de inserção político pedagógico-social (11).

Na perspectiva deste estudo a Enfermagem é potencializadora do sujeito, pois como profissão de sensibilidade e subjetividade humana, mostra-se mais próxima e com agudez de perceber a necessidade do outro de modo a contribuir para que este possa identificá-la e prover esforços à modificação do que for necessário; repadronizar o que se mostra passível de sê-lo feito e retorquir o que produz danos à saúde, portanto, percorrer o itinerário de relações dialógicas, de respeito aos modos de vida, cultura valores e crenças como nos ensina Maidelene Leininger (12).

\section{MÉTODO}

Estudo desenvolvido na transversalidade com o método misto, no qual foram considerados 50 e 50\% para ambas às análises avaliativo-interpretativa e compreensiva dos dados (13).

Na primeira etapa, utilizou-se um desenho de estudo do tipo pesquisa-ação assentado na concepção problematizadora e complexificadora do projeto guarda-chuva: "Educação em saúde no enlace diagnóstico preventivo do pé-diabético: oficinas educativas na abordagem proximal no cuidado à saúde", cuja premissa teórica enovela a práxis interdisciplinar -Autocuidado e Diversidade Cultural-, no 
direcionamento de um cuidado individualizado e integral, sem perder de vista a globalidade dos sistemas - individuo-família e comunidade, tranversalizando a abordagem dos saberes da resiliência como potencialidade teórica. Aprovado pelo CEP/UESB - protocolo $n^{\circ} .247 .938$, seguindo a Resolução ${ }^{\circ}$. 466/2012 (14), do Departamento de Saúde II, da Universidade Estadual do Sudoeste da Bahia, campus Jequié, BA.

Ao delinear pela estratégia pesquisa-ação buscou-se unir a pesquisa à práxis na aproximação de saberes de modo a favorecer a promoção de mudanças ao bem-estar coletivo das pessoas. Tratou-se de ir ao encontro dos ensinamentos de Paim et al. (15). A natureza do estudo foi delineada também no enlace da abordagem descritiva (15), a qual possibilitou observar, registrar, analisar e inter-relacionar os acontecimentos sem manipulá-los em todas as etapas do estudo.

Este foi realizado no município de Jequié, BA, no período de agosto/2013-julho/2014, no Núcleo Interdisciplinar de Estudos e Extensão em Cuidados à Saúde da Família em Convibilidade com Doenças Crônicas (NIEFAM), sendo as participantes 13 pessoas com idade entre 43 a 76 anos, média $=61,62$ anos, todas do sexo feminino, segundo os critérios de inclusão: ter diagnóstico de DM2; estar cadastrada no NIEFAM e Unidades de Saúde da Família de Jequié, BA; ter participado em mais de $70 \%$ das oficinas de educação em saúde do projeto guarda-chuva supracitado $^{2}$; não apresentar déficit cognitivo que

\footnotetext{
${ }^{2}$ As atividades do projeto guarda-chuva consistiram em investigar os pés de pessoas com diagnóstico de DM2 cadastradas no NIEFAM, visando identificar sinais e sintomas da Neuropatia Periférica (NP), ou seja, a complicação crônica mais comum e mais incapacitante do diabetes; avaliar seu grau de conhecimento sobre a NP; promover ações interventivas de educação em saúde à promoção da saúde dos pés e reavaliar o seu grau de conhecimento após abordagens intervencionista. Desenvolvido através de oficinas de educação em saúde sobre NP, semanalmente, de natureza problematizadora-construtivista, por um período quatro meses e duração de 90 minutos cada atividade, com avaliação e reavaliação continuada da estratégia utilizada pela equipe de pesquisadores.
}

impossibilitasse de participar apreendendo das oficinas e de responder ao questionário de coleta de dados do estudo; e, ser anuente a participar da pesquisa, assinando Termo de Consentimento Livre e Esclarecido e Termo de Autorização de uso de Imagens e Depoimentos. As participantes foram identificadas, segundo a ordem numérica de realização do questionário de coleta de dados (Exp.: Participante 01 ). $\mathrm{O}$ fechamento amostral foi por saturação dos dados, assim, se deu a partir do momento em que as informações fornecidas pouco acrescentaram ao material obtido (16).

Os instrumentos de coletas de dados consistiram de um questionário elaborado com 11 questões enoveladoras do saber/ conhecer os cuidados de si das participantes, sobre a saúde dos pés diante da patologia DM, alicerçado no Consenso Internacional sobre Diabetes - IDF (17), e nos conteúdos programáticos de abordagem educativoproblematizadora desenvolvidos no projeto guarda-chuva. Outro instrumento foi um roteiro sistematizado para observação, das participantes nas oficinas educativas de cuidadoà saúde, pela equipe de pesquisadores. Este instrumento visou à transversalidade de olhares, percepção das pesquisadoras, sobre os comportamentos e questionamentos das participantes referentes ao saber que detinham e construíam no evoluir das oficinas educativo-interativas. Tratou-se de ir-se desenovelando a abordagem pesquisaação à análise compreensiva dos dados.

O questionário fora realizado de forma individual, em local reservado e no qual as perguntas foram respondidas segundo os temas: Estou fazendo; Faço parcialmente; Faço raramente e Não faço. Estes foram realizados imediatamente seis meses após o término das oficinas, constituindo a etapa 2 do estudo. Destaca-se que a elaboração do questionário, além de assentar-se no IDF (17) também foi subsidiada por outra pesquisa realizada no ano 2013, intitulada: "Pé-diabético - conhecimento de pessoas 
com Diabetes Mellitus"3, a qual buscou identificar o nível de conhecimento das pessoas com DM. Este também se abriu em um leque de três outros desenhos metodológicos, a exemplo o que ora se apresenta, o qual se encaminhou por um estudo nos prontuários dos participantes do NIEFAM, no levantamento de dados sociodemográfico e de estado de saúde, a guisa de entremear os dados emergidos das pesquisas supracitadas corroborativos a esta.

Os documentos procedentes dos dados da pesquisa compõem o banco de informações do NIEFAM/UESB, arquivados em local de acesso restrito e exclusivo aos pesquisadores do núcleo.

A análise dos dados foi desenvolvida utilizando a perspectiva interpretativo-compreensiva sugerida por Miles e Huberman (18). Tratou-se de por em ação um modelo interativo de análise: redução dos dados, na qual ocorreu a simplificação, abstração e sua transformação; em seguida a apresentação, de tal modo a possibilitar que se tirassem conclusões preliminares; e, por fim, o delineamento e verificação da conclusão, com a identificação de padrões possíveis de explicações e configurações. Assentou-se no olhar sensível e avaliativo ao que emergiu dos temas dos questionários, dos olhares/ percepção da equipe de pesquisadores ao instrumento de observação e das oficinas educativas de cuidado à saúde ao encontro da pesquisa-ação.

Para a análise quantitativa dos dados, fez-se uso da estatística descritiva para determinar os valores dado em porcentagem para as variáveis nominais e média e desvio padrão para as variáveis categóricas. Para isso, foi utilizado o programa estatístico Statistical Package for Social Science - SPSS (versão 15.0, Chicago, IL, EUA).

${ }^{3}$ Hora FL. Pé diabético - conhecimento de pessoas com diabetes mellitus [monografia]. [Jequié]: Universidade Estadual do Sudoeste da Bahia; 2013. 53 p.

\section{RESULTADOS}

A média de idade das participantes desta pesquisa foi de $61,62( \pm 8,26)$ anos; a maioria casada $(61,54 \%)$; se consideram pardas (46,15\%), católicas $(53,85 \%)$; com o ensino fundamental incompleto $(69,23 \%)$; donas de casa $(53,85 \%)$. Referente à renda familiar (46,15\%) preferiu não responder e 46,15\% respondeu perceber entre 1 a 2 salários mínimos mensais (Tabela 1).

Com relação às características das idosas no que se refere à saúde, a maioria $(53,85 \%)$ apresenta sobrepeso, Índice de Massa Corporal $(\text { IMC })^{4}$ médio de $27,70( \pm 5,69) \mathrm{kg} / \mathrm{m}^{2}$. Do total, 10 apresentaram valores fora do recomendado pela World Health Organization (19); a totalidade (100\%) informou realização de atividade física, destas (92,31\%) 3 vezes na semana de forma regular; $(61,54 \%)$ realizar dieta prescrita por um profissional de saúde.

Com relação à doença, as participantes tiveram diagnóstico de DM $(23,08 \%)$ a menos de 1 ano, e $(76,92 \%)$ a mais de 1 ano, $(92,31 \%)$ informaram utilizar hipoglicemiante oral para controle glicêmico e apenas uma $(7,76 \%)$ fazer uso de insulinoterapia, assim como apresentar complicação macrovascular.

Outra doença de impacto na vida das participantes refere-se à Hipertensão Arterial Sistêmica (HAS) em que $(84,62 \%)$ tem diagnóstico, sendo que $(81,82 \%)$ a mais de 1 ano, fazendo uso de tratamento anti-hipertensivo (76,92\%), e conjugado dos tipos de fármacos (60,00\%).

Destaca-se que como tratamento complementar para os desvios de saúde $(84,62 \%)$ informaram fazer uso de fitoterápicos, exclusivamente para DM $(36,36 \%)$ (Tabela 2$)$.

\footnotetext{
${ }^{4} \mathrm{O}$ IMC foi uma variável observada em conformidade a Associação Brasileira para o Estudo da Obesidade e da Síndrome Metabólica - ABESO, na observância de que a obesidade está fortemente associada a risco e agravo potencial ao Diabetes mellitus do tipo 2, entre outras.
} 
A Tabela 3 compreende a fase 2 do estudo de revisitar os saberes das participantes após etapa das oficinas educativas na abordagem do cuidado dos pés. Deste modo, no que se refere aos cuidados específicos com os pés $(76,9 \%)$ faz exames dos pés semanalmente com uso de espelhos; (100\%) informaram não fazer "escalda pés"; $(84,6 \%)$ hidratação dos pés; (100\%) secar entre os dedos dos pés e não usar creme nestes espaços; (76,9\%) não fazem uso de meia de algodão sem costura; (100\%) não removem calos com lixas ou produtos químicos e também não cortam unhas rente ao dedo ou arredonda, sendo que $(46,2 \%)$ fazem retirada de cutícula; e, (100\%) fazem verificação do calçado antes de usá-lo (Tabela 3).

Tabela 1. Descrição das variáveis socioeconômicas. Jequié, BA. 2014.

\begin{tabular}{|c|c|c|}
\hline Variáveis Socioeconômicas & Quantidade & Porcentagem \\
\hline \multicolumn{3}{|l|}{ Estado Civil } \\
\hline Casada & 8 & $61,54 \%$ \\
\hline Viúva & 2 & $15,38 \%$ \\
\hline Divorciada & 2 & $15,38 \%$ \\
\hline Solteira & 1 & $7,69 \%$ \\
\hline \multicolumn{3}{|l|}{ Raça / Etnia } \\
\hline Branca & 3 & $23,08 \%$ \\
\hline Parda & 6 & $46,15 \%$ \\
\hline Negra & 4 & $30,77 \%$ \\
\hline \multicolumn{3}{|l|}{ Religião } \\
\hline Católica & 7 & $53,85 \%$ \\
\hline Protestante & 6 & $46,15 \%$ \\
\hline \multicolumn{3}{|l|}{ Escolaridade } \\
\hline Ensino superior completo & 1 & $7,69 \%$ \\
\hline Ensino médio completo & 2 & $15,38 \%$ \\
\hline Ensino Fundamental incompleto & 9 & $69,23 \%$ \\
\hline Analfabetismo & 1 & $7,69 \%$ \\
\hline \multicolumn{3}{|l|}{ Profissão } \\
\hline Do lar & 7 & $53,85 \%$ \\
\hline Aposentada & 2 & $15,38 \%$ \\
\hline Enfermeira & 1 & $7,69 \%$ \\
\hline Técnica de enfermagem & 1 & $7,69 \%$ \\
\hline Cuidadora / Aposentada & 1 & $7,69 \%$ \\
\hline Servente & 1 & $7,69 \%$ \\
\hline \multicolumn{3}{|c|}{ Renda familiar com base no salário nínimo $(\mathrm{R} \$ 678,00)^{\star}$} \\
\hline$\leq 1$ salário mínimo & 1 & $7,69 \%$ \\
\hline$>1$ e $\leq 2$ salários mínimo & 4 & $30,77 \%$ \\
\hline$>2$ salários mínimos & 2 & $15,38 \%$ \\
\hline Não informou & 6 & $46,15 \%$ \\
\hline
\end{tabular}

${ }^{*}$ Renda familiar com base no salário nínimo 2013. 
Tabela 2. Descrição das variáveis relacionadas à doença. Jequié, BA. 2014.

\begin{tabular}{|c|c|c|}
\hline Variáveis relacionadas à doença & Quantidade & Porcentagem \\
\hline \multicolumn{3}{|l|}{ Índice de Massa Corporal } \\
\hline Obesidade Grau 2 & 2 & $15,38 \%$ \\
\hline Obesidade Grau 1 & 1 & $7,69 \%$ \\
\hline Sobrepeso & 7 & $53,85 \%$ \\
\hline Padrão Normal & 1 & $7,69 \%$ \\
\hline Abaixo do Peso & 1 & $7,69 \%$ \\
\hline Não informado & 1 & $7,69 \%$ \\
\hline \multicolumn{3}{|l|}{ Prática de Atividade Física } \\
\hline $\operatorname{Sim}$ ( 3 vezes na semana) & 12 & $92,31 \%$ \\
\hline $\operatorname{Sim}(>3$ vezes na semana $)$ & 1 & $7,69 \%$ \\
\hline \multicolumn{3}{|c|}{ Realiza dieta alimentar prescrita por algum profissional } \\
\hline Sim & 8 & $61,54 \%$ \\
\hline Não & 3 & $23,08 \%$ \\
\hline Às vezes & 2 & $15,38 \%$ \\
\hline \multicolumn{3}{|c|}{ Tempo de diagnóstico de DM (anos) } \\
\hline$\leq 1$ ano & 3 & $23,08 \%$ \\
\hline$>1$ ano $\mathrm{e} \leq 10$ anos & 5 & 38.46 \\
\hline$>10$ anos & 5 & 38.46 \\
\hline \multicolumn{3}{|l|}{ Hipoglicemiante VO } \\
\hline Sim & 12 & $92,31 \%$ \\
\hline 1 tipo & 6 & $50,00 \%$ \\
\hline 2 tipos & 4 & $33,33 \%$ \\
\hline 3 tipos & 1 & $8,33 \%$ \\
\hline 4 tipos & 1 & $8,33 \%$ \\
\hline Não & 1 & $7,69 \%$ \\
\hline \multicolumn{3}{|l|}{ Insulina } \\
\hline $\operatorname{Sim}(\mathrm{NPH})$ & 1 & $7,69 \%$ \\
\hline Não & 12 & $92,31 \%$ \\
\hline \multicolumn{3}{|l|}{ Complicações } \\
\hline Sim (macrovascular) & 1 & $7,69 \%$ \\
\hline Não & 12 & $92,31 \%$ \\
\hline \multicolumn{3}{|l|}{ Tem HAS } \\
\hline Sim & 11 & $84,62 \%$ \\
\hline$\leq 1$ ano & 1 & $9,09 \%$ \\
\hline$>1$ ano e $\leq 10$ anos & 7 & $63,64 \%$ \\
\hline$>10$ anos & 2 & $18,18 \%$ \\
\hline Não sabe & 1 & $9,09 \%$ \\
\hline Não & 2 & $15,38 \%$ \\
\hline \multicolumn{3}{|l|}{ Anti-hipertensivo } \\
\hline Sim & 10 & $76,92 \%$ \\
\hline 1 tipo & 2 & $20,00 \%$ \\
\hline 2 tipos & 6 & $60,00 \%$ \\
\hline 3 tipos & 2 & $20,00 \%$ \\
\hline Não & 1 & $7,69 \%$ \\
\hline Não Informado & 2 & $15,38 \%$ \\
\hline \multicolumn{3}{|l|}{ Fitoterápicos } \\
\hline Sim & 11 & $84,62 \%$ \\
\hline $\mathrm{DM}$ & 4 & $36,36 \%$ \\
\hline Outros & 7 & $63,64 \%$ \\
\hline Não & 2 & $15,38 \%$ \\
\hline
\end{tabular}


Tabela 3. Revisitando os saberes das usuárias do NIEFAM, da etapa 2, dos cuidados dos pés. Jequié, BA. 2014.

\begin{tabular}{lcccc}
\hline & $\begin{array}{c}\text { Estou Fa- } \\
\text { zendo }(\%)\end{array}$ & $\begin{array}{c}\text { Faço } \\
\text { Parcialmente } \\
(\%)\end{array}$ & $\begin{array}{c}\text { Faço } \\
\text { Raramente } \\
(\%)\end{array}$ & $\begin{array}{c}\text { Não } \\
\text { Faço } \\
(\%)\end{array}$ \\
\hline Temas & 76,9 & 0 & 0 & 23,1 \\
Faz exame dos pés? & 0 & 0 & 0 & 100 \\
Faz "escalda-pés"? & 84,6 & 0 & 7,7 & 7,7 \\
Faz hidratação dos pés? & 0 & 0 & 0 & 100 \\
Faz uso de creme entre os dedos dos pés? & 100 & 0 & 0 & 0 \\
Seca entre dos dedos dos pés? & 23,1 & 0 & 0 & 76,9 \\
Faz uso de meia de algodão sem costura? & 0 & 0 & 0 & 100 \\
Faz remoção de calos com lixas ou produtos químicos? & 0 & 0 & 0 & 100 \\
Faz corte das unhas de modo rente ao dedo e redonda? & 46,2 & 7,7 & 7,7 & 38,5 \\
Faz retirada de cutícula? & 100 & 0 & 0 & 0 \\
Faz verificação do calçado antes de usá-lo? & 30,8 & 0 & 0 & 69,2 \\
Faz uso de sabonete neutro para lavar os pés?
\end{tabular}

\section{DISCUSSÃO E CONCLUSÃO}

As variáveis sociodemográficas deste estudo encontram aderência na literatura (3, 5). Em destaque, o estado conjugal, como aquele com influência no cuidado da terapêutica do DM no domicílio de forma contributiva pelo cônjuge de modo que a relação intradomiciliar harmoniosa, na maioria das vezes, favorece os cuidados com a doença (3). Dado observado neste estudo na verbalização das participantes sobre o processo de viver cotidiano com DM, em que o cônjuge mostrava-se vigilante e atento à dieta alimentar familiar na atenção de sua esposa. Na maioria, elas disseram cozinhar para a toda a família sem separação de tipo alimentar. Neste particular, o cuidado domiciliar enredava os coabitantes do lar ao cuidar preventivamente de sua saúde.

Referente à escolaridade houve predomínio do ensino fundamental incompleto, cor da pele manifestada ser na maioria negra e parda e trabalhadora do lar, com renda familiar de até dois salários mínimos. Esses da- dos vão ao encontro do que é difundido pelo Instituto Brasileiro de Geografia e Estatística (20) e de uma população em que pardos e pretos constituem o maior contingente de pessoas de baixa escolaridade e em maior risco de vulnerabilidade social. Sobre isto, destaca-se que a baixa escolaridade caminha na contramão da justiça social e do fim das discriminações sociais e disparidades socioeconômicas, sendo a faixa etária igual e superior a 60 anos, a com maior percentual nacional $26,6 \%$, a exemplo do que identificamos neste estudo, que encontra aderência em outra pesquisa no âmbito do município no qual foi realizada esta pesquisa, sendo estudados 117 idosos com idade mínima de 60 anos e máxima de 106 anos, com média de 80,79 ( \pm 9,77 ) anos, verificando-se maior frequência de idosos do sexo feminino $(70,09 \%)$, não alfabetizados $(63,25 \%)$, e com renda familiar de 1 a 3 salários mínimos (53,80\%) (21). Estes achados também se refletem em outro estudo realizado na região nordeste do Brasil, sendo constatadas taxas elevadas de analfabetismo entre os idosos; perto de 65 em cada 100 que não sabiam ler nem escrever (22). 
Esta realidade não é exclusividade da região Nordeste, pois estudo realizado em São Carlos, SP, cuja amostra foi de 523 participantes, $61,4 \%$ de idosos nas faixas etária de 60 a 69 anos, 50,0\% era analfabeto. E destes, $62,0 \%$ de mulheres analfabetas (23).

Estes dados corroboram com nosso estudo, e nos remete a questão de gênero, muito embora, este não seja o foco desta pesquisa, mas vale salientar em observância aos direitos constitucionalmente adquiridos, os quais não fazem discriminação entre homens e mulheres. A desigualdade de gênero constitui uma das grandes contradições da sociedade que se mantém ao longo da história da civilização e tem colocado as mulheres em um lugar social de subordinação e de violação dos direitos humanos (24).

Do exposto, o somatório dessas variáveis demonstra o grau de vulnerabilidade dessas pessoas aos cuidados de si, por déficit educacional, valorização da autoimagem e forma de aquisição de bens de consumo e material, fatores corroborativos de uma sociedade ainda discriminatória de visão míope assentada na invisibilidade social (25).

Ainda, o fato de todas apresentarem uma religião, direciona o raciocínio para a valorização pessoal-social direcionada pelo acolhimento de grupos de pertencimento religiosos. Este raciocínio encontra-se ancorado no estudo de Valla (26), em que o autor chama atenção para a tentativa das classes em resolver problemas de ordem material com a finalidade de viver de modo pleno, buscando na religiosidade o apoio social que não é encontrado nos órgãos de direito e o alívio do sofrimento e do direcionamento na busca de conforto social; tanto no sentido temporário físico e mental, quanto no sentido de redução do estresse que castiga determinado órgão do corpo (26).

Evidenciamos ainda no estudo, o sobrepeso e a obesidade das participantes avaliada pelo IMC. Neste caso, o aumento no peso das pessoas com DM é um fator preponderante para aumentar o risco de de- senvolvimento do pé-diabético (27). Assim, uma dieta alimentar, para além da restrição de alimentos ricos em glicose e lipídios, agregam valores positivos ao tratamento e prevenção do pé-diabético $(5,28,29)$. E este controle glicêmico na prevenção de complicações nos pés também tem sido reconhecido por pessoas com DM (29), caso que se repetiu no NIEFAM.

Além disso, os resultados indicaram pessoas praticantes de atividade física regular (mais que 3 vezes na semana). Sabe-se que complementarmente à dieta alimentar, a prática regular de atividade física controla/reduz os índices glicêmicos e diminui o sobrepeso e a obesidade $(3,5,28,29)$. Ainda, aliar essas duas ações também reduz a necessidade do uso de hipoglicemiantes e o risco de doença cardiovascular $(2,4)$.

Sobre o tempo do diagnóstico, com participantes do NIEFAM com mais de 10 anos de DM direciona-se para a importância de redobrar o cuidado com o pé, tendo em vista que o aumento no tempo do diagnóstico aumenta o risco de surgimento de complicações $(3,5,6,29)$. Além disso, com a chegada da velhice há singularidades como dificuldade de controle glicêmico e, como consequência, menor eficácia na prevenção das complicações macrovasculares; riscos de crises hipoglicêmicas graves e maior intensidade e frequência de efeitos colaterais dos hipoglicemiantes orais (4). Dado observado em nosso estudo em que uma das participantes já apresentava complicação macrovascular.

$\mathrm{Na}$ Tabela 3 podemos observar que os comportamentos de risco quanto ao desconhecimento ou em conhecendo não realizar o cuidado referente aos pés foram de 23,1\% das participantes não faz exame dos pés e $46,2 \%$ faz retirada de cutículas. As demais variáveis demonstram que a maioria das participantes realiza cuidados com os pés.

O exame dos pés constitui um meio de prevenção e detecção precoce de risco de neuropatia diabética. Trata-se de uma precaução referida na literatura em muitas pes- 
quisas sobre boas práticas de cuidados com os pés, as quais incluem: inspeção diária; realização de exercícios para os pés; não andar descalço; uso de sapatos adequados, macios e confortáveis; verificação do espaço interno dos sapatos antes de usá-los; uso de meias de algodão sem costura; corte adequado das unhas; não utilizar bolsa de água quente ou escalda-pés; secar os espaços interdigitais (5, 28,29 ).

A adoção de medidas preventivas a saúde dos pés das participantes deste estudo indica a capacidade de elas terem compreendido os saberes desenvolvidos nas oficinas educativas do projeto guarda-chuva na fase 1 . Neste tocante, desenvolveram fator de proteção para cuidado com os pés, e, consequentemente, prevenção de comorbidades pelo DM. Este dado encontra eco na pesquisa de Bragança e colaboradores, desenvolvida em uma Unidade Básica de Saúde, no interior de São Paulo, em que 92\% dos entrevistados relataram ter recebido algum tipo de informação sobre DM. Destes, $71 \%$ informaram que a unidade de saúde oferecia atividades educativas sobre a patologia, apesar de somente $46 \%$ disserem ter participado dessas atividades. No montante dos que participaram, 45 pessoas informaram que as atividades desenvolvidas as auxiliaram no controle da doença, dieta, tratamento medicamentoso, insulinoterapia, autocuidado e inovações para o tratamento (5).

Contrariamente aos cuidados de promoção a saúde na Atenção Básica, identificamos na literatura estudos em que os resultados apontaram para uma minoria de profissionais de saúde voltados à ação preventiva da neuropatia diabética, pois, não examinavam os pés dos usuários do serviço. Assim, não desenvolvia na prática a profilaxia preventiva, nem tão pouco, ações de educação continuada com vistas à prevenção de agravos a saúde das pessoas (29). Este dado é preocupante, pois as pessoas que são acometidas por lesões nos pés decorrente do DM, na maioria das vezes, apresentam outras complicações associadas, o que requer atenção profissional ao estar-se diante de uma pessoa com DM, para que o olhar cuidativo seja multidimensional (30).

A importância do acompanhamento multiprofissional já é perspectivada nos cuidados as pessoas em condição crônica (31), mas, infelizmente, na realidade de saúde pública brasileira mostra-se como um desafio a ser superado. A inexistência deste tem favorecido a perpetuação de um ciclo de patologias, que, por vezes, tem se tornado comum em nossa sociedade, sendo ainda mais agravante nas classes menos favorecidas.

No direcionamento do acompanhamento supracitado, foi realizado um estudo no ambulatório de um Hospital Universitário do Norte do Estado do Paraná, para avaliar um programa de cuidados com os pés de pessoas com DM. Neste, foi observado que ações cuidativas desenvolvidas foram significantes no estado de prevenção de complicações por DM dos usuários do serviço. Tais participantes avaliaram como de maior relevância as ações de orientações do programa sobre o autocuidado com os pés, $84 \%$; verificação da sensibilidade dos pés, $65,3 \%$; e cuidados com corte das unhas e micoses $49,3 \%$. Dos usuários $89,3 \%$ informaram melhora no aspecto dos pés após aprendizado (32).

A educação em saúde é enfatizada como abordagem potencializadora dos cuidados da pessoa com DM, em destaque, aos fatores de risco, capazes de desencadear complicações no processo saúde-doença (6). Neste particular, o profissional de saúde tem papel fundamental na realização de práticas que aliem educação em saúde, treinamento e sistematização do autocuidado (7). As práticas de prevenção do pé diabético requerem ações diretas, simples e aliadas à capacitação profissional, devendo-se direcionar em via de mão-dupla -as dimensões pessoa-família-comunidade na perspectiva da potencialidade das pessoas para o cuidado de si, o que se mostra como coresponsabilização, autocuidado, ao alcance do êxito nos resultados esperados (7).

O DM é capaz de desencadear proces- 
sos complexificadores ao viver humano das pessoas com impacto na qualidade de vida, sendo um grave problema de ordem social e pública no Brasil.

O olhar para essa evidência chama atenção para estratégias de abordagem às potencialidades humana para o autocuidado. Neste direcionamento, saber-conhecer sobre as pessoas e o seu processo de viver-convivendo com a doença mostra-se como condição precípua a elaboração de estratégias eficientes e eficazes de controle e tratamento da doença. Nesta pesquisa, este foi o direcionamento tomado no desenovelamento de estudos em um projeto guarda-chuva alicerçador do saber-conhecer proximal ao cuidado de pessoas com DM.

A abordagem adotada permitiu conhecer o perfil das participantes da pesquisa e os seus saberes sobre sua condição crônica, os quais, alicerçadores do direcionamento dos caminhos seguidos à potencialização destas ao autocuidado, na estratégia pesquisa-ação e na (re)avaliação do percurso tomado, de modo que os resultados evidenciaram que a estratégia contribuiu à reflexão-ação à medidas protetivas no cuidado com os pés.

Salienta-se neste estudo, a relevância da participação das pessoas no planejamento e gestão dos cuidados de sua saúde em grupos de pertencimento, cujas ações viabilizem estratégias de educação em saúde assentadas em pressupostos construcionista em uma metodologia proximal que alie educação em saúde, treinamento e sistematização do autocuidado a melhoria da qualidade de saúde das pessoas.

\section{REFERÊNCIAS}

1. Schmidt MI, Duncan BB, Azevedo e Silva G, Menezes AM, Monteiro CA, Barreto $\mathrm{SM}$, et al. Chronic non-communicable diseases in Brazil: burden and current challenges. Lancet. 2011; 377(9781): 1949-61.

2. Ministério da Saúde. Estratégias para o cuidado da pessoa com doença crônica: diabetes mellitus [Internet]. Brasília: Ministério da Saúde (BR), Secretaria de Atenção à Saúde, Departamento de Atenção Básica; 2013 [citado 06 ago 2013]. 160 p. Cadernos de Atenção Básica, nº 36. Disponível em: http://bvsms.saude.gov. br/bvs/publicacoes/estrategias_cuidado_ pessoa_diabetes_mellitus_cab36.pdf .

3. Amaral AS, Tavares DMS. Cuidados com os pés: conhecimento entre pessoas com diabetes mellitus Rev Eletr Enf [Internet]. 2009 [citado 10 set 2013]; 11(4): 801-10. Disponível em: http://www.fen.ufg.br/ revista/v11/n4/pdf/v11n4a05.pdf.

4. Sociedade Brasileira de Diabetes. Diretrizes da Sociedade Brasileira de Diabetes 2009 [Internet]. $3^{a}$ ed. Itapevi, SP: A. Araújo Silva Farmacêutica; 2009 [citado 06 agosto 2013]. 400 p. Disponível em: http://dms.ufpel.edu.br/ares/bitstream/handle/123456789/270/11\%20 \%20\%202009\%20diretrizes\%20diabete. pdf? sequence $=1$.

5. Bragança CM, Gomes IC, Fonseca MRCC, Colmanetti MNS, Vieira MG, Souza MFM. Avaliação das práticas preventivas do pé diabético. J Health Sci Inst. 2010; 28(2): 159-63.

6. Cisneros LL. Avaliação de um programa para prevenção de úlceras neuropáticas em portadores de diabetes. Rev Bras Fisioter. 2010; 14(1): 31-7.

7. Manheze AIB, Pezzutto TM. Diabetes e risco de pé diabético: importância do autocuidado. CuidArte Enfermagem [Internet]. 2011 [citado 06 ago 2013]; 5(2): 137-42. Disponível em: http:// fundacaopadrealbino.org.br/facfipa/ ner/pdf/CuidArte\%20Enfermagem $\% 20$ v.\%205,\%20n.\%202,\%20jul.-dez.\%20 2011.pdf.

8. Renpenning KM, Taylor SG. Self-Care 
Theory in Nursing: Selected Papers of Dorothea Orem. New York: Springer Publishing Company; 2003. 374 p.

9. Spichler ER, Spichler D, Lessa I, Costa e Forti A, Franco LJ, LaPorte RE. Capturerecapture method to estimate lower extremity amputation rates in Rio de Janeiro, Brazil. Rev Panam Salud Publica. 2001; 10(5): 334-40.

10. The Global Lower Extremity Amputation Study Group. Epidemiology of lower extremity amputation in centres in Europe, North America and East Asia. Br J Surg. 2000; 87(3): 328-37.

11. Lei de Diretrizes e Bases da Educação Nacional (BR). Lei 9.394/96 [Internet]. Brasília: Imprensa Oficial, Diário Oficial, v. 134, n. 248, 1996 [citado 06 ago 2013]. Disponível em: http://www.planalto.gov. br/ccivil_03/leis/19394.htm

12. Leininger MM, Mcfarland MR. Culture Care Diversity and Universality. A Worldwide Nursing Theory. $2^{\text {nd }}$ ed. Sudbury, Massachusetts: Jones and Bartlett Publishers; 2006. 413 p.

13. Creswell JW. Projeto de pesquisa: Métodos qualitativo, quantitativo e misto. $3^{a}$ ed. Porto Alegre: Artmed, 2010.296 p.

14. Ministério da Saúde. Diretrizes e normas regulamentadoras sobre pesquisa envolvendo seres humanos [Internet]. Resolução 466. 2012. Brasília: Conselho Nacional de Saúde (BR); 2012 [citado 06 ago 2013]. Disponível em: http://bvsms. saude.gov.br/bvs/saudelegis/cns/2013/ res0466_12_12_2012.html>.

15. Paim L, Trentini M, Madureira VSF, Stamm M. Pesquisa convergente-assistencial e sua aplicação em cenários da Enfermagem. Cogitare Enferm. 2008; 13(3): 380-6.

16. Denzin NK, Lincoln YS. The SAGE Handbook of qualitative research. $3^{\text {rd }}$ ed. London: SAGE Publications, Inc; 2005. $1210 \mathrm{p}$.

17. Grupo de Trabalho Internacional sobre Pé
Diabético. Consenso Internacional sobre Pé Diabético/ publicado sob a direção de Hermelinda Cordeiro Pedrosa; tradução de Ana Claudia de Andrade, Hermelinda Cordeiro Pedrosa [Internet]. Brasília: Secretaria de Estado de Saúde do Distrito Federal; 2001 [citado 06 agosto 2013]. 100 p. Disponível em: http://189.28.128.100/ dab/docs/publicacoes/geral/conce_inter_ pediabetico.pdf

18. Miles MB, Huberman AM. Qualitative data analysis: an expanded sourcebook. $2^{\text {nd }}$ ed. London: SAGE Publications, Inc; 1994.

19. World Health Organization. Global Strategy on Diet, Physical Activity and Health. What is overweight and obesity? [Internet]. Geneva: WHO; 2014 [citado 30 dez 2014]. Disponível em: http://www. who.int/dietphysicalactivity/childhood_ what/en

20. Brasil. Ministério do Planejamento, Orçamento e Gestão. Instituto Brasileiro de Geografia e Estatística-IBGE. Censo Demográfico 2010. Características gerais da população, regiões e pessoas com deficiência [Internet]. Rio de Janeiro: IBGE; 2012 [citado 17 jul 2014]. Disponível em: http://biblioteca.ibge.gov.br/visualizacao/periodicos/94/cd_2010_religiao_deficiencia.pdf

21. Torres GV, Reis LA, Reis LA, Fernandes $\mathrm{MH}$, Xavier TT. Relação entre funcionalidade familiar e capacidade funcional de idosos dependentes no município de Jequié (BA). Rev Baiana Saúde Pública. 2010; 34(1): 19-30.

22. Guimarães LHCT, Galdino DCA, Martins FLM, Abreu SR, Lima M, Vitorino DFM. Avaliação da capacidade funcional de idosos em tratamento fisioterapêutico. Revista Neurociências. 2004; 12(3): 13033.

23. Feliciano AB, Moraes AS, Freitas ICM. O perfil do idoso de baixa renda no Município de São Carlos, São Paulo, 
Brasil: um estudo epidemiológico. Cad Saude Publica. 2004; 20(6): 1575-85.

24. Guedes RN, Silva ATMC, Fonseca RMGS. A violência de gênero e o processo saúde-doença das mulheres. Esc Anna Nery Rev Enferm. 2009; 13(3): 625-31.

25. Costa VF, Constantino ML. Invisibilidade social: outra forma de preconceito [Internet]. Brasil: Barrinha, SP; 2007 [citado 17 jul 2014]. Disponível em: http://www. overmundo.com.br/overblog/invisibilidade-social-outra-forma-de-preconceito.

26. Valla VV. Pobreza, emoção e saúde: uma discussão sobre pentecostalismo e saúde no Brasil. Rev Bras Educ [Internet]. 2002 [citado 17 jul 2014]; 19: 63-75. Disponível em: http://www.scielo.br/pdf/rbedu/n19/ n19a05.pdf

27. Nehring P, Mrozikiewicz-Rakowska B, Krzyżewska M, Sobczyk-Kopcioł A, Płoski R, Broda G, et al. Diabetic foot risk factors in type 2 diabetes patients: a crosssectional case control study. J Diabetes Metab Disord. 2014; 13: 79.
28. Rocha RM, Zanetti ML, Santos MA. Comportamento e conhecimento: fundamentos para prevenção do pé diabético. Acta Paul Enferm. 2009; 22(1): 17-23.

29. Andrade NHS, Mendes KDS, Faria HTG, Martins TA, Santos MA, Teixeira CRS, et al. Pacientes com diabetes mellitus: cuidados e prevenção do pé diabético em atenção primária à saúde. Rev Enferm UERJ. 2010; 18(4): 616-21.

30. Duarte N, Gonçalves A. Pé diabético. Angiologia e Cirurgia Vascular. 2011; 7(2): 65-79.

31. Barros MFA, Mendes JC, Nascimento JA, Carvalho AGC. Impacto de intervenção fisioterapêutica na prevenção do pé diabético. Fisioter Mov. 2012; 25(4): 74757.

32. Silva EC, Haddad MCL, Rossaneis MA. Avaliação de um programa sistematizado de cuidados com os pés na perspectiva dos pacientes com diabetes mellitus. UNOPAR Cient. Ciênc Biol Saude. 2013; 15(1): 21-5. 